AC 2007-1708: TEACHING ENGINEERING CONCEPTS ACROSS MULTIPLE DISCIPLINES USING A SINGLE PLATFORM BASED ON VIRTUAL INSTRUMENTATION

Shekhar Sharad, National Instruments 


\title{
Teaching Engineering Concepts Across Multiple Disciplines Using a Single Platform based on Virtual Instrumentation
}

\begin{abstract}
Multidisciplinary Labs are becoming increasingly important with convergence of technologies. Continuing budget cuts make it difficult to fund new infrastructure such as physical labs and alternatives that enable educators to reuse existing labs are needed. With the evolution of the desktop and graphical design technologies based on Virtual Instrumentation, it is now possible to implement multidisciplinary labs that span different facets of engineering from control and signal processing to embedded design, from chemistry and physics to electrical and computer engineering. In this paper, we will explain how Virtual Instrumentation helps to establish multidisciplinary labs. We will also examine a modular, cost-effective, laboratory platform, NI ELVIS (Educational Laboratory Virtual Instrumentation Suite) from National Instruments that has gained acceptance in academia as platform to teach concepts in sensors \& transducers, circuits, electronics, microcontroller programming, control, signal processing and embedded design and test.
\end{abstract}

\section{Introduction}

The recent years have seen an increase in emphasis on laboratories. Innovative educators are implementing hands-on learning across multiple disciplines. Whether it is courses in sciences such as physics or chemistry or in electrical and biomedical engineering, there is a significant increase in need for laboratories. While this demand is increasing at a rapid pace, the infrastructure is not increasing at the same pace. This has led educators to seek out innovative techniques to leverage the existing infrastructure to better deliver a hands-on learning environment by setting up multi-disciplinary labs. In this paper, we will outline one of the most promising methodologies, Virtual Instrumentation and how, with recent innovations, it can help educators setup multidisciplinary labs. We will also explore an innovative laboratory platform, NI Educational Laboratory Virtual Instrumentation Suite or the NI ELVIS that has gained acceptance in over 1500 universities worldwide as an instrumentation platform that can serve the needs of multiple disciplines.

\section{Background on Virtual Instrumentation}

The objective of this paper is not to educate the audience on Virtual Instrumentation as this has been effectively done in literature[12,13,14,15]. Rather, the objective of this paper is to examine how the recent advances in Virtual Instrumentation can enable educators to leverage this technology to setup multidisciplinary labs. Virtual Instrumentation platforms such as NI LabVIEW have evolved to enable educators to not just teach how to acquire data from sensors and instruments but be able to teach signal processing and control design concepts as well as mechatronics and embedded design. Educators at Rose-Hulman Institute of Technology[1] have used Virtual Instrumentation that is based on graphical programming to teach signal processing concepts. Educators at Rensselaer Polytechnic Institute (RPI) Mechatronics [2] have used Virtual Instrumentation to teach mechatronics concepts and design innovative systems such as the HOT$\mathrm{V}$ (Human Object Transport Vehicle) in one semester from concept to prototype. An interesting demonstration of this system can be found at [3]. Another project in the same department at RPI is the levitation system that is created using NI ELVIS. Educators at Georgia Tech in their ECE 
3041 [4] course are using Virtual Instrumentation and NI ELVIS to teach circuit design and instrumentation concepts. Another example of the evolution of Virtual Instrumentation to enable design is from University of Texas at Austin [5] where educators are using Virtual Instruments to teach communication system design concepts. While the exploration of all of the innovative techniques that have advanced Virtual Instrumentation is beyond the scope of the paper, the authors attempt to outline some options that will gives educators a possibility outside of the conventional techniques that enable them to take advantage of existing infrastructure and instrumentation as well as reuse the same lab space for labs from other disciplines.

\section{Virtual Instrumentation and Multidisciplinary Education}

As outlined in the previous section, the evolution of Virtual Instrumentation has opened up a possibility to leverage this platform for setting up multidisciplinary labs. What is being proposed in this paper is an example implementation of what the authors feel would be one way that educators can leverage this evolution in Virtual Instrumentation. Before outlining this example, it is noteworthy to point out that while Virtual Instrumentation has evolved, it does not exclude students from learning using traditional instrumentation. In fact, the authors believe that it augments this approach and a simple search on the internet can show that Virtual Instrumentation provides numerous interfaces to communicate with most traditional instrumentation systems used in laboratories. An example is the instrument driver network from National Instruments[6]. Additionally, with industry consortiums such as the PXI Alliance [7], Virtual Instrumentation has gained industry acceptance and hence has a fair probability that it will be used by students upon graduation.

\section{An Example Multidisciplinary Lab Setup}

Table 1 shows some labs that multiple disciplines share and some of the hardware and

Table 1. Example Setups for Labs that may be Shared by Multiple Disciplines

\begin{tabular}{|l|l|l|}
\hline Labs & $\begin{array}{l}\text { Example } \\
\text { Hardware/Instruments }\end{array}$ & \multicolumn{1}{c|}{ Example Software } \\
\hline Instrumentation & $\begin{array}{l}\text { Scope, Func. Gen, VPS, } \\
\text { Different Sensors }\end{array}$ & $\begin{array}{l}\text { Optional, C, Visual Basic, } \\
\text { custom }\end{array}$ \\
\hline Circuit Design & Breadboard, Scope, Func. Gen & SPICE Simulation software \\
\hline Control Design/Mechatronics & FPGA/MPU based boards & LabVIEW, Xilinx tools \\
\hline Signal \& Image Processing & Optional, webcams, DSPs & LabVIEW, TI CCS \\
\hline Communication Design & RF Up/down converters & Custom \\
\hline
\end{tabular}

software that educators may use. Note that there are multiple software packages that are available that can cater to these different areas and only some of the more common ones are referred to. The key to note in the above setup - first, there are different hardware platforms that are needed. Hardware takes up space and hence is something that must be taken into consideration. Let us now see how Virtual Instrumentation can be applied to simplify the setups in table 1. Table 2 shows an example lab setup, which is now implemented with Virtual Instrumentation. For the purposes of this paper, we have chosen NI LabVIEW as our Virtual Instrumentation software. We have also used two hardware platforms, PXI (PCI eXtensions for Instrumentation)[8], a modular instrumentation standard that is being used in research labs and 
industries and NI ELVIS[9]. Table 2 also shows an example of which University uses this kind of a system to teach a particular lab.

Table 2. An Example Multidisciplinary Lab Setup with Virtual Instrumentation

\begin{tabular}{|l|l|l|l|}
\hline \multicolumn{1}{|c|}{ Labs } & \multicolumn{1}{|c|}{ Hardware } & \multicolumn{1}{c|}{ Software } & \multicolumn{1}{c|}{ Example University } \\
\hline Instrumentation & $\begin{array}{l}\text { PXI System and } \\
\text { NI ELVIS; Traditional } \\
\text { Instruments }\end{array}$ & LabVIEW & $\begin{array}{l}\text { UT Austin (Biomedical } \\
\text { Instr.) }\end{array}$ \\
\hline Circuit Design & $\begin{array}{l}\text { NI ELVIS; Traditional } \\
\text { Instruments }\end{array}$ & LabVIEW, Multisim & $\begin{array}{l}\text { Univ. of Connecticut } \\
\text { (Circuit Design) }\end{array}$ \\
\hline $\begin{array}{l}\text { Control Design } \\
\text { Mechatronics }\end{array}$ & $\begin{array}{l}\text { PXI with FPGA plugin, } \\
\text { NI ELVIS with ADI } \\
\text { BF533/537, NI ELVIS } \\
\text { with Quanser boards, } \\
\text { FPGA boards }\end{array}$ & $\begin{array}{l}\text { LabVIEW, Xilinx } \\
\text { Tools, Visual } \\
\text { DSP++ }\end{array}$ & RPI (Mechatronics), \\
\hline $\begin{array}{l}\text { Signal \& Image } \\
\text { Processing }\end{array}$ & $\begin{array}{l}\text { NI ELVIS with DSP } \\
\text { plugin boards, TI DSP }\end{array}$ & $\begin{array}{l}\text { LabVIEW, C, C++, } \\
\text { TI Code Composer } \\
\text { Studio }\end{array}$ & $\begin{array}{l}\text { Univ of Connecticut } \\
\text { (Biomedical Engr); } \\
\text { Rose-Hulman Institute } \\
\text { of Technology }\end{array}$ \\
\hline $\begin{array}{l}\text { Communication } \\
\text { Design }\end{array}$ & PXI System & $\begin{array}{l}\text { LabVIEW, Custom, } \\
\text { C, C++ }\end{array}$ & $\begin{array}{l}\text { UT Austin (MIMO } \\
\text { Systems) }\end{array}$ \\
\hline
\end{tabular}

From Table 2, there are several things that are worth noting. Virtual Instrumentation does not need educators to abandon and eliminate the current lab setups. They can continue using the existing software and hardware with the modified setup because Virtual Instrumentation provides interfaces for connecting to multiple buses and devices. Additionally, with the evolution of Virtual Instrumentation software, it can now be applied in labs that include control and design concepts providing educators an avenue to have software that is economical and can be shared across multiple disciplines. The hardware implementation allows for flexibility also - NI ELVIS is an education platform while the PXI-based systems provide high-end instrumentation in a compact form factor (a single 3U 8-slot PXI chassis can hold the equivalent of 6 traditional instruments in the space of one). It should also be noted that this is an example implementation of Virtual Instrumentation for Multidisciplinary labs and is open to innovation. There could also be numerous permutations of the same setup depending upon the individual constraints.

\section{NI ELVIS for Multidisciplinary Labs}

One of the more commonly found Virtual Instrumentation platforms for academia is the NI ELVIS (Educational Laboratory Virtual Instrumentation Suite). More information about this hardware platform including the individual components and the hardware specifications can be found on the National Instruments website[10]. Additionally, there is abundant literature that report on using NI ELVIS for learning and teaching concepts[16,17,18] 


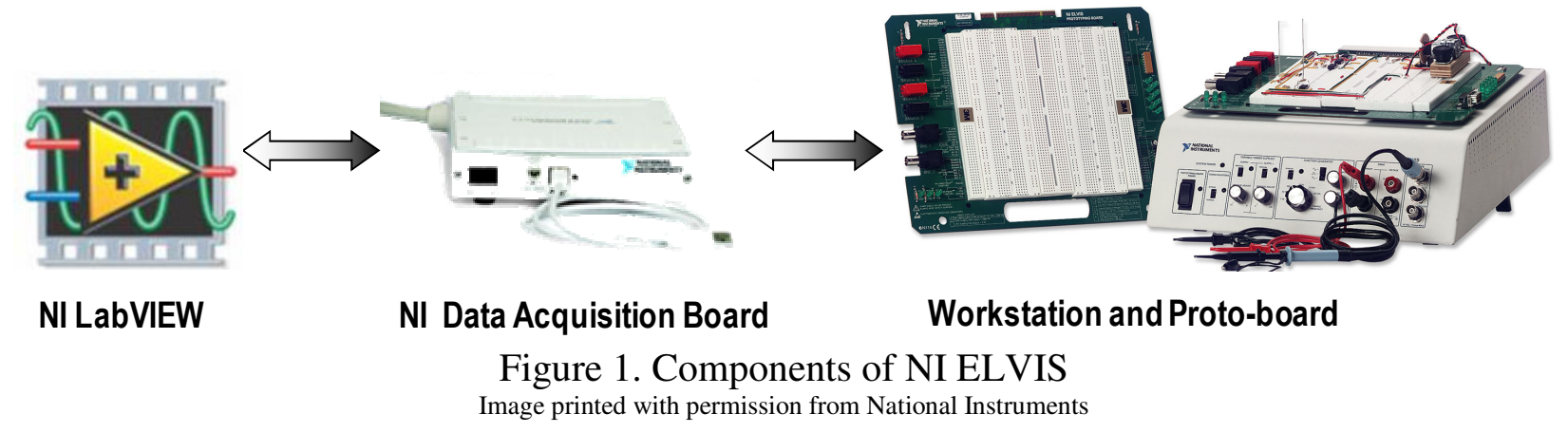

Figure 1 shows the different components of NI ELVIS. The key differentiator for the NI ELVIS that makes it a candidate for Multidisciplinary labs is its integrated instrumentation suite. NI ELVIS includes some of the most commonly used laboratory instruments. Table 3 shows the list of these instruments included with NI ELVIS. More details about these instruments can be found on the National Instruments website.

Table 3. NI ELVIS Instruments

\begin{tabular}{|l|l|}
\hline 1 & Digital Multimeter (DMM) \\
\hline 2 & Oscilloscope \\
\hline 3 & Function Generator \\
\hline 4 & Variable Power Supplies (VPS) \\
\hline 5 & Bode Analyzer \\
\hline 6 & Arbitrary Waveform Analyzer \\
\hline 7 & Dynamic Signal Analyzer \\
\hline 8 & Digital Reader \\
\hline 9 & Digital Writer \\
\hline 10 & Impedance Analyzer \\
\hline 11 & Two-wire Current Analyzer \\
\hline 12 & Three-wire Current Analyzer \\
\hline
\end{tabular}

For the purposes of this paper, the authors focus briefly on how the NI ELVIS can be used while teaching different labs.

\subsection{Circuit Design}

University of Connecticut is an example of an Educational Institution that uses NI ELVIS to teach circuit design with NI Multisim and LabVIEW. NI ELVIS includes a prototyping board that students can use to build circuits. In addition, with NI LabVIEW, educators can compare saved SPICE simulations with real-world data. An extensive and in-depth case study for circuit design with NI Multisim, NI LabVIEW and NI ELVIS[11] is available online for interested readers.

\subsection{Instrumentation, Sensors and Transducers}

University of Texas at Austin uses NI ELVIS to teach Biomedical engineering students to work with sensors and transducers. NI ELVIS uses the NI DAQ driver technology which helps acquire data from different sensors such as: 
- Thermocouple, thermistor, RTDs

- Strain Gauges

- Load Cells

- Pressure Transducers

- Photodiodes

- Vernier sensors (with Vernier Adapters as shown in figure 2)

- Pasco sensors (with Pasco Adapters) and others

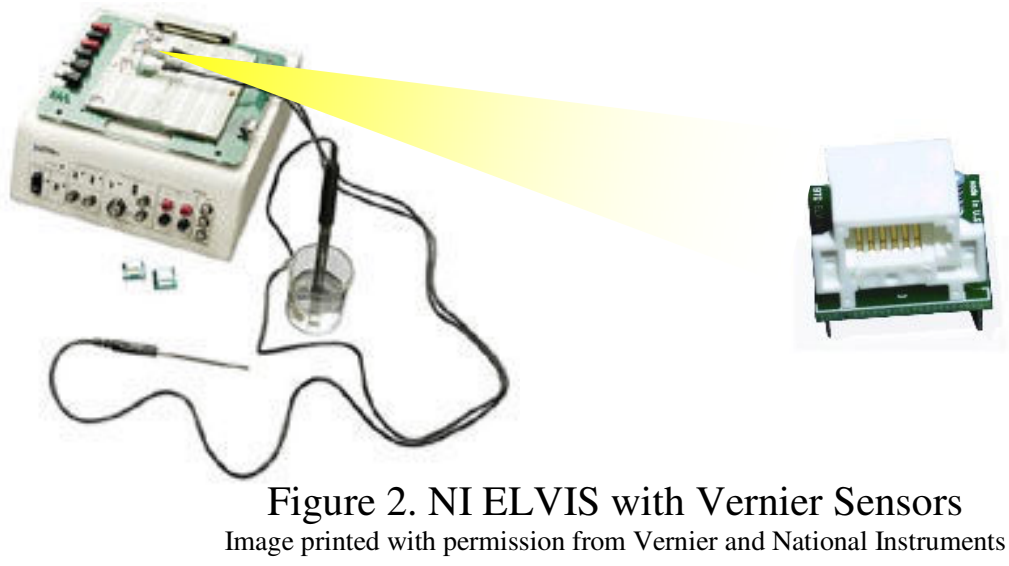

\subsection{Microcontroller Education with Freescale HCS12 \& NI ELVIS}

Eastern Washington University has recently setup a microcontroller lab that uses the Freescale MCUSLK HCS12 teaching platform and NI ELVIS. While NI ELVIS is not used to program the microcontroller board, it is used to test all the parameters such as operating frequency and voltage. The Freescale MCUSLK includes an NI ELVIS connector that can be used to leverage the integrated instruments of NI ELVIS instead of using dedicated instruments

\subsection{Embedded Labs with ADI Blackfin BF533/537 and NI ELVIS}

The ADI Blackfin BF533/537 boards include an NI ELVIS connector. A recent technological development is the ability of LabVIEW to target the ADI Blackfin boards directly making it more accessible to non-EE and non-embedded students. Coupled with the integrated instrumentation functionality of NI ELVIS, it provides a cost-effective platform (see next section) for a programming and measurement system.

\section{Cost Comparison for NI ELVIS Vs Traditional Instrumentation Systems}

The authors performed a cost analysis of NI ELVIS with a leading manufacturer of traditional instrumentation. The results are tabulated in table 4 . For the purposes of this study, the authors only did a price analysis for the 4 most commonly used instruments. Instruments such as the Impedance analyzers cost $\$ 21,430$ (lowest) from the same manufacturer - this high cost can be attributed to the research-lab type specifications. Such equipment may be compared against the PXI systems that offer the same specifications for a lower cost - but that cost analysis is beyond the scope of this paper. Interested readers are invited to perform a cost analysis for the remaining instruments by visiting the websites of leading traditional instrument vendors and comparing them with PXI as well as NI ELVIS platforms. 
Table 4. Cost Comparison Between NI ELVIS and Standalone Instruments

\begin{tabular}{|l|l|l|}
\hline Instrument & Stand-alone Instrument & NI ELVIS \\
\hline Oscilloscope & $\$ 1100$ & Included \\
\hline DMM & $\$ 399$ & Included \\
\hline Function Generator & $\$ 1853$ & Included \\
\hline Variable Power Supply & $\$ 1236$ & Included \\
\hline & & \\
\hline & & \\
\hline Total & $\$ 4588(4$ instruments $)$ & $\begin{array}{l}\$ 1999(12 \text { instruments, PCI) } \\
\text { \$2699 (12 instruments, USB) }\end{array}$ \\
\hline Difference (\%) & & $56 \%$ less (PCI) \\
& & $41 \%$ less (USB) \\
\hline
\end{tabular}

*Stand-alone instruments are from Agilent and pricing for all the above instruments can be found online

It should also be noted that the above cost analysis is for one laboratory. If multiple labs had to be setup, the costs may change. NI ELVIS as outlined in the paper can be used for multiple labs, and may be able to bring costs down.

\section{Conclusion}

Multidisciplinary labs are a cornerstone of future engineering and science education. With the increased emphasis on hands-on learning, ABET accreditation criteria and need for low-cost, reusable, state-of-the-art instrumentation, novel methodologies to reuse existing infrastructure is needed. One methodology that helps achieve these goals is Virtual Instrumentation. In this paper, we show an example multidisciplinary course setup with Virtual Instrumentation. We also looked at NI ELVIS, an educational platform based on Virtual Instrumentation that has gained acceptance throughout academia in more than 1500 universities worldwide. We also explored how the integrated instruments in NI ELVIS help educators teach classes in different domains such as circuits and instrumentation. In summary, this paper attempts to give educators an alternative to traditional instrumentation that is low-cost and reusable and is based on the recent innovations in Virtual Instrumentation and show some examples of the same.

\section{References}

[1] “A Study of Graphical Vs Textual Programming for Teaching DSPs", M Yoder, B Black 2006 ASEE Annual Conference \& Exposition

[2] Mechatronics at RPI, http://mechatronics.rpi.edu

[3] Human Object Transport Vehicle Video, http://mechatronics.rpi.edu/video.htm

[4] ECE 3041 Course website at Georgia Tech, http://users.ece.gatech.edu/ tbrewer/ece3041/

[5] Wireless Communications Lab at UT Austin, http://users.ece.utexas.edu/ rheath/courses/wirelesslab/index.php 
[6] National Instruments Instrument Driver Network, http://www.ni.com/devzone/idnet/

[7] PXI Systems Alliance, http://www.pxisa.org/

[8] Official PXI specifications from PXI Systems Alliance, http://www.pxisa.org/specs.htm

[9] NI ELVIS resources, http://www.ni.com/academic/ni_elvis/

[10] Hardware Specifications for NI ELVIS, http://www.ni.com/pdf/products/us/ni_elvis.pdf

[11] The NI Electronics Education Platform: A Case Study,

http://zone.ni.com/devzone/cda/tut/p/id/3004

[12] H Goldberg, "What is virtual instrumentation?", Instrumentation \& Measurement Magazine, IEEE 2000

[13] MG Vose, G Williams, “Laboratory virtual instrument engineering workbench”, BYTE, 1986

[14] M Parten, "Using Virtual Instruments in a Measurements Laboratory", Proceedings of ASEE 2003 Annual Conference

[15] AH Taner, NM White, "Virtual instrumentation: a solution to the problem of design complexity in intelligent instruments", Measurement+ Control, 1996

[16] J Del Rio, E Trullols, A Manuel, J Mendez, ELVIS. A new tool for teaching and training Instrumentation and Measurement Technology Conference, IEEE 2004

[17] J Ventura, R Drake, J McGrory, NI ELVIS has entered the lab [educational laboratory virtual instrumentation suite], SoutheastCon, 2005. Proceedings. IEEE, 2005

[18] T Eppes, P Schuyler, T Oruganti, Pilot Test Results of a New Distance Laboratory Platform, ASEE Annual Conference \& Exposition, 2005 